\title{
Consequences of duration of untreated psychosis (DUP) on peripheral vascular endothelial function: A Pilot Study
}

M. Pilecki ${ }^{1}$, S. Chłopicki², W. Krzyściak ${ }^{3}$, E. Szczęsny - Małysiak ${ }^{2}$, A. Kij² ${ }^{2}$ P.Gawęda ${ }^{4}$, M. Szwajca ${ }^{1}$, N. Ś Smierciak $^{1}$

${ }^{1}$ Department of Child and Adolescent Psychiatry, the Jagiellonian University, Medical College, Krakow, Poland; ${ }^{2}$ Jagiellonian Centre for Experimental Therapeutics, the Jagiellonian University, Medical College, Krakow, Poland; 3JDepartment of Medical Diagnostics, Faculty of Pharmacy, the Jagiellonian University, Medical College,Krakow,Poland; ${ }^{4}$ Department of Clinical Biochemistry, the Jagiellonian University, Medical College, Krakow, Poland;

JAGIELLONIAN UNIVERSITY MEDICAL COLLEGE

\section{O B J E C T I V E}

Among patients suffering from schizophrenia, the most common cause of premature deaths are cardiovascular disorders. Development of atherosclerotic plaque leading to cardiovascular episodes occurs only in the case of the cooccurrence of endothelial dysfunction of the blood vessels. According to our knowledge there have been no papers evaluating the output endothelial status prior to the onset of post-psychotic treatment.

\section{MA TER I A L A N D M THO D S}

Evaluation of peripheral vascular endothelial status was conducted by the analysis of concentration of the nitric oxide metabolite $\left(\mathrm{NO}_{2}^{-}\right)$in plasma. The risk of atherosclerotic plaque was assessed through concentration of monocytes in plasma. Concentration of nitric oxide metabolite and monocytes were collected in plasma in 15 first-episode psychosis patients: 9 women and 6 men (median age: 26 years; range: 15 to 35 years) before the onset of antipsychotic treatment. The DUP was assessed.

\section{R E SU L T S}

With the increase in the length of DUP, the lower the $\mathrm{NO}_{2}^{-}$ concentration and the higher the concentration of monocytes.

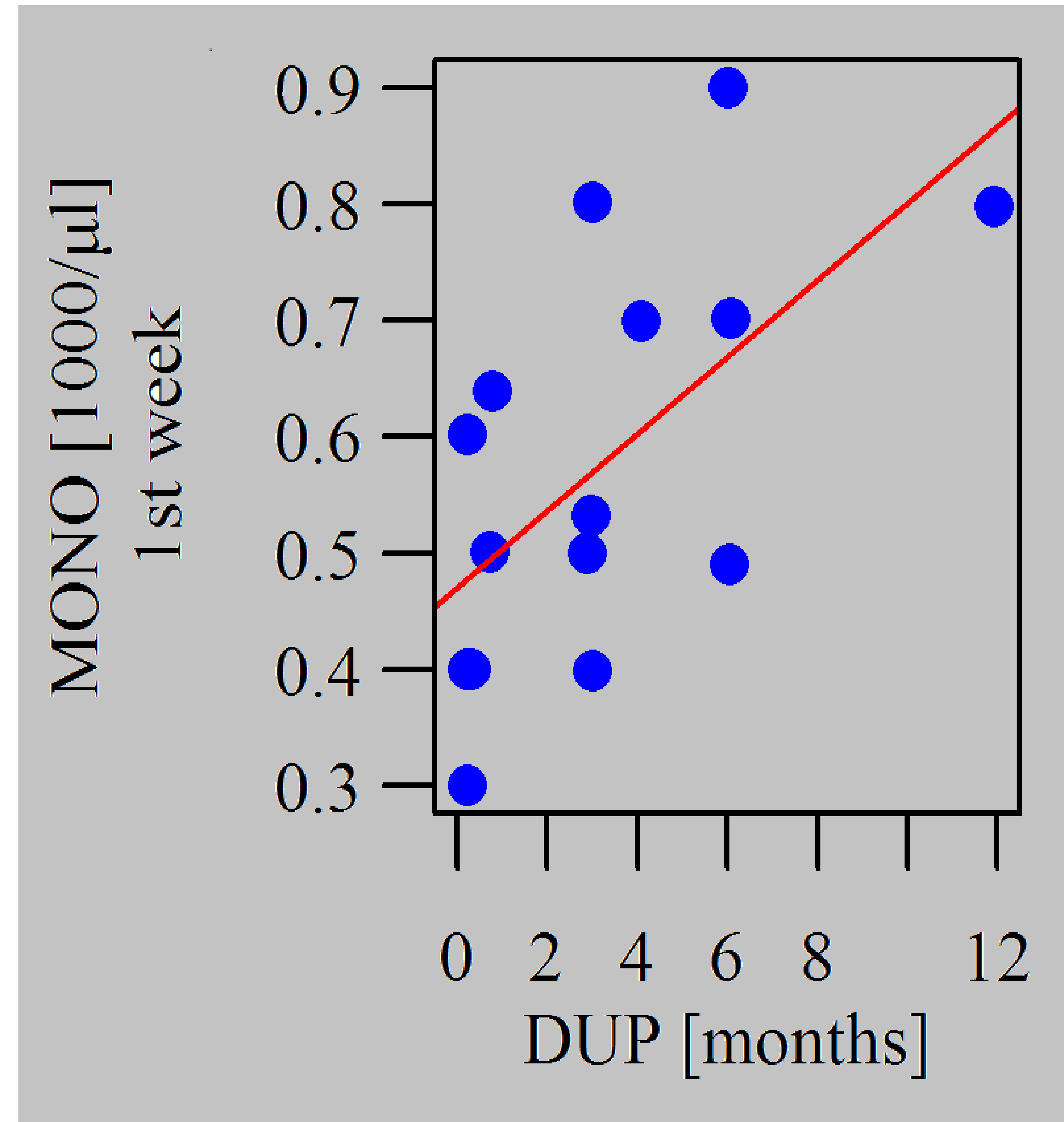

$\Rightarrow$ The longer the duration of untreated psychosis, the worse the state of peripheral vascular endothelium.

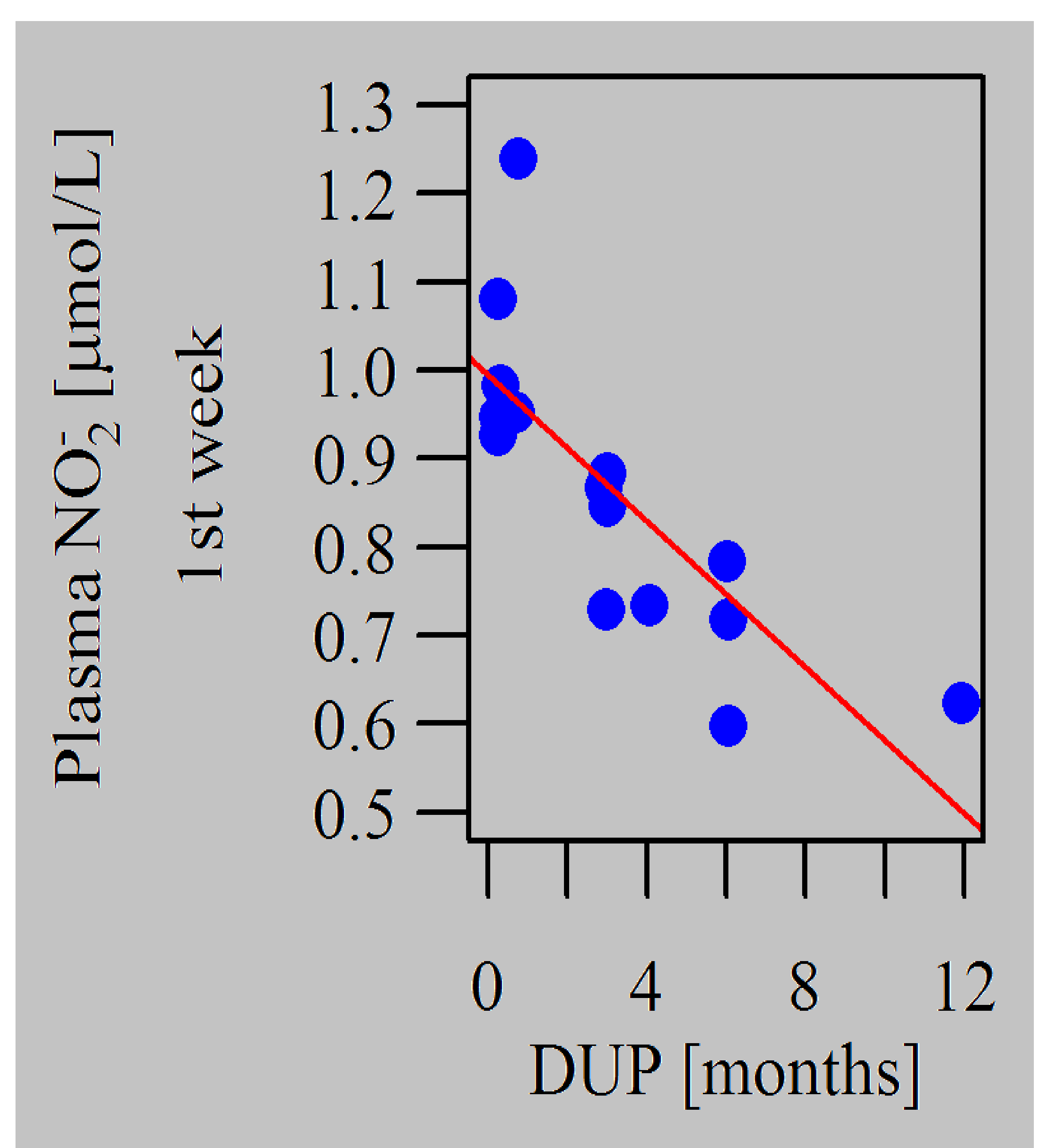

\section{O N C L US I O N S}

Results should be taken cautiously due to the small sample size but show that the longer the duration of untreated psychosis without antipsychotic treatment, the more severe deterioration of peripheral endothelial function occurs, thus increases the risk of the cardiovascular diseases. 